\title{
Frustrated packing of trees
}

\section{Entropic Effects in Cell Lineage Tree Packings}

Authors: Jasmin Imran Alsous, Paul Villoutreix, Norbert Stoop, Stanislav Y.

Shvartsman, and Jörn Dunkel

Nature Physics, v. 14, n. 10, p. 1016 - 1021, 2018

\section{Recommended with a Commentary by Alexander Y. Grosberg, Department of Physics and Center for Soft Matter Research, New York University, 726 Broadway, New York, NY 10011}

The main discovery of the paper by Imran Alsous et al is that spatial arrangement of cells in the forming fruit fly egg is to a large extent governed by simple geometrical constraints. The purpose of my comment is much more narrow, I want to attract attention to the connection of their results with some seemingly unrelated problems in soft matter and polymer physics.

In brief, the picture considered by Imran Alsous et al is as follows. In the initial stages of egg production, a tree-like structure of 16 cells is formed from a single stem cell by a series of (incomplete) divisions. Of these 16, one will eventually become the egg, while 15 others play some supporting role (unlike the usual biological terminology, incomprehensible for a non-professional, these supporting cells are called nurse cells). The divisions are incomplete in the sense that each daughter cell remains connected to its mother cell by a tiny tube, called ring canal, through which presumably nurse cells can supply nutrients to the future egg. Through the series of these incomplete divisions, a structure arises with a tree-like topology. Moreover, this tree reflects the history of cell divisions and thus represents the cell lineage tree (which is why it is called CLT). For a better description of this process interested reader is referred to either the original article by Imran Alsous et al, or an accompanying $\mathrm{N} \& \mathrm{~V}[1]$.

Imran Alsous et al pointed out that this tree-like structure, consisting of cells with a significant (excluded) volume, must be embedded in 3D and placed in a limited space on the internal surface of a convex shaped domain. They discovered that topologically different trees can be packed in different numbers of ways, interpreted this as difference in packing entropy, and argued that this entropic effect is significant for the real system in question.

As stated, my goal is to point out parallels to some other problems. To begin with, consider a dendrimer - a regular branched polymer with one three-functional root, 3 threefunctional branched points of generation one, $3 \cdot 2^{1}$ three-functional points of generation two, $\ldots, 3 \cdot 2^{\ell-1}$ three-functional points of generation $\ell$, and $3 \cdot 2^{\ell-1}$ of single-functional ends, or "leaves" (see, e.g., [2]). The well known (and obvious) fact is that large generation dendrimers cannot fit in space: assuming bond lengths are fixed, the maximal size of an 
$\ell$-generations dendrimer grows only linearly with $\ell$, while the number of units $N=3 \cdot 2^{\ell}-2$ is exponential in $\ell$. Other branched polymers, not so aggressively branching as dendrimers, can still experience frustrations.

One way to think about it mathematically is to consider a branched polymer with some $N=K^{3}$ monomers, with all bonds of a fixed length 1 , on a cubic lattice in 3D obeying the excluded volume constraints (such that no two monomers can occupy the same site), and try to place it within $K \times K \times K$ cube. Similar problem for a linear polymer is well known (see, e.g., [3] for the latest results). Quite clearly, branching will impose very significant frustrations, but (for this writer, at least) it is an open question how to characterize them.

The problem, besides its mathematical interest, may have also a practical implication. Many viruses have RNA molecules as their genome (influenza virus, painfully familiar to all of us, is one example). By self-pairing, a large RNA molecules can form effectively a branched polymer. In part, secondary structures of these RNAs can rearrange to ease packing frustrations to some degree (see, e.g., $[4,5]$ ). However, it is not entirely clear to which extent secondary structure, or tree-branching topology, can rearrange, or can behave as annealed, and to which extent it is fixed, or quenched. If it is quenched, then we face a problem rather similar to that examined by Imran Alsous and co-authors, except it is not clear whether packing is inside the 3D body, or on the (internal) surface of the capsid [6].

In physics, we all cherish beautiful physical analogies - between pendulum and $R L C$ contour, or between electrostatics, diffusion, and low Reynolds number hydrodynamics, for example. Do such beautiful analogies exist also in biological physics?

\section{References}

[1] Ben D. MacArthur, "Connected development", Nature Physics, v. 14, n. 10, p. 975 976, 2018.

[2] David Boris, and Michael Rubinstein, "A Self-Consistent Mean Field Model of a Starburst Dendrimer: Dense Core vs Dense Shell", Macromolecules, v. 29, n. 22, p. 7251-7260, 1996.

[3] Raoul D. Schram, and Helmut Schiessel "Exact Enumeration of Hamiltonian Walks on the $4 \times 4 \times 4$ " Cube and Applications to Protein Folding" J. Phys. A: Math. Theor., v. 46, 485001, 2013.

[4] Aron M. Yoffe, Peter Prinsen, Ajaykumar Gopal, Charles M. Knobler, William M. Gelbart, and Avinoam Ben-Shaul "Predicting the sizes of large RNA molecules," Proc. Natl. Ac. Sci. USA, 105, n. 42, p. 16153-16158, 2008.

[5] Joshua Kelly, Alexander Y. Grosberg, and Robijn Bruinsma "Sequence Dependence of Viral RNA Encapsidation," J. Phys. Chem. B, v. 120, n. 26, p. 6038 - 6050, 2016.

[6] Peter G. Stockley, and Reidun Twarock (editors) "Emerging Topics in Physical Virology," World Scientific (Singapoor), 2010. 